\title{
Pinning control of fractional-order weighted complex networks
}

\author{
Yang Tang, ${ }^{1, a)}$ Zidong Wang, ${ }^{1,2}$ and Jian-an Fang ${ }^{1, b)}$ \\ ${ }^{1}$ College of Information Science Technology, Donghua University, Shanghai 201620, China \\ ${ }^{2}$ Department of Information Systems and Computing, Brunel University, \\ Uxbridge, Middlesex, UB8 3PH, United Kingdom
}

(Received 18 October 2008; accepted 17 December 2008; published online 10 February 2009)

\begin{abstract}
In this paper, we consider the pinning control problem of fractional-order weighted complex dynamical networks. The well-studied integer-order complex networks are the special cases of the fractional-order ones. The network model considered can represent both directed and undirected weighted networks. First, based on the eigenvalue analysis and fractional-order stability theory, some local stability properties of such pinned fractional-order networks are derived and the valid stability regions are estimated. A surprising finding is that the fractional-order complex networks can stabilize itself by reducing the fractional-order $q$ without pinning any node. Second, numerical algorithms for fractional-order complex networks are introduced in detail. Finally, numerical simulations in scale-free complex networks are provided to show that the smaller fractional-order $q$, the larger control gain matrix $D$, the larger tunable weight parameter $\beta$, the larger overall coupling strength $c$, the more capacity that the pinning scheme may possess to enhance the control performance of fractional-order complex networks. (C) 2009 American Institute of Physics.
\end{abstract}

[DOI: $10.1063 / 1.3068350$ ]

Recently, fractional-order differential systems have been widely investigated due to their potential applications in viscoelasticity, dielectric polarization, quantum evolution of complex systems, and many other fields. On the other hand, research of complex networks has triggered tremendous interest during the past decade. Most studies to date have concerned integer-order complex networks. In this paper, we consider the pinning control problem of fractional-order weighted complex dynamical networks. The fractional-order complex networks generalize wellstudied integer-order complex networks. Some local stability properties of such pinned fractional-order networks are derived and the valid stability regions are estimated by utilizing the eigenvalue analysis and fractional-order stability theory. A surprising finding that the fractional-order networks can stabilize itself by reducing the fractional-order $q$ without pinning any node is presented. The numerical algorithms for fractional-order networks are also presented. In the end, computer simulations in scale-free networks are given to show that the smaller fractional-order $q$, the larger control gain matrix $D$, the larger tunable weight parameter $\beta$, the larger coupling strength $c$, the more capacity that the pinning strategy can possess to accelerate the control rate of fractional-order networks.

\section{INTRODUCTION}

Many large-scale systems in nature and human societies, such as, genetic regulatory networks, food webs, the internet, scientific citation web, etc., can be modeled by networks, where nodes are individuals of the system and the edges

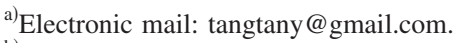

${ }^{b)}$ Electronic mail: jafang@dhu.edu.cn.
}

represent interactions between them. ${ }^{1-4}$ One decade ago, to describe the transition from regular networks to random ones, Watts and Strogatz (WS) (Ref. 1) introduced the concept of small-world networks. After that, a scale-free network model was proposed by Barabási and Albert (BA), ${ }^{2}$ in which the degree distribution of the nodes follows a powerlaw form. Thereafter, the small-world features and the scalefree properties of complex networks have attracted increasing attention from researchers in their studies. ${ }^{5-8}$

On the other hand, in recent years, fractional calculus has drawn much attention due to its application in physics and engineering. ${ }^{9-12}$ It has been revealed that, in interdisciplinary fields, various systems have been found to exhibit fractional dynamics. For example, viscoelasticity, dielectric polarization, quantum evolution of complex system, fractional kinetics, and anomalous attenuation can be described by fractional differential equations. ${ }^{9-12}$ Meanwhile, it has been shown that many chaotic systems display the fractionalorder chaotic dynamics, such as, fractional-order Lorenz system, ${ }^{13}$ fractional-order Chua's system, ${ }^{14}$ fractional-order cellular neural networks, ${ }^{15}$ fractional-order Rössler system, ${ }^{16}$ and so on.

Recently, the collective dynamics analysis of complex networks has led to a host of interesting effects. ${ }^{6-8,17-34}$ In particular, the study on controlling the dynamics of a network and guiding it to a desired state, such as, an equilibrium point or a periodic orbit of the network has become an interesting and important direction in this research field. ${ }^{7,18-20,22-24,26,27}$ It has been revealed that, in the process of controlling various networks, feedback control serves as a simple and effective approach for stabilization and synchronization. However, it is widely believed that it is impossible to add controllers to all nodes. To reduce the control cost, some feedback injections may be added to a 
fraction of network nodes, which is known as pinning control. $^{17-20,22-24,26,27,33}$ In Ref. 17, pinning control of spatiotemporal chaos was discussed. In Ref. 7, both specific and random pinning schemes were studied. Li et al. ${ }^{18}$ investigated the pinning control of complex dynamical networks to their equilibriums including both the random networks and the scale-free networks. However, to the best of the authors' knowledge, the research on the dynamical analysis of complex networks, such as, control and synchronization of complex networks, has mainly focused on integer-order complex networks, and the corresponding research on fractional-order complex networks has received very little attention despite its practical significance.

Motivated by the above observations, four seemingly natural questions arise as follows: (1) Does the fractionalorder complex networks exist which can be pinned? (2) What kind of pinning controllers may be designed to ensure a fractional-order complex network is stabilized to its equilibriums? (3) How large of the coupling strength should be used for a fractional-order network with a fixed structure to achieve local stability? (4) What factors effectively affect the convergence rate of controlling the fractional-order networks?

The overall aim of this paper is to provide convincing answers to the above four questions by providing a rather general framework. The rest of this paper is organized as follows: In Sec. II, some preliminaries of fractional calculus and fractional-order complex networks are briefly outlined. The main results for pinning control on fractional-order complex networks are given in Sec. III. In Sec. IV, the algorithms for simulating fractional-order networks and numerical examples are provided. Concluding remarks are presented in Sec. V.

\section{PRELIMINARIES}

There are many definitions of fractional derivative. The most well-known definition is the Riemann-Liouville fractional derivative, defined by

$$
D^{q} f(t)=\frac{d^{m} J^{m-q} f(t)}{d t^{m}}, \quad q>0,
$$

where $m=\lceil q\rceil$, i.e., $m$ is the first integer which is not less than $q . J^{\gamma}$ is the $\gamma$-order Riemann-Liouville integral operator which can be described by the following expression:

$$
J^{\gamma} g(t)=\frac{\int_{0}^{t}(t-\tau)^{\gamma-1} g(\tau) d \tau}{\Gamma(\gamma)}, \quad \gamma>0
$$

where $\Gamma(\cdot)$ is a gamma function given by

$$
\Gamma(z)=\int_{0}^{\infty} t^{z-1} e^{-t} d t
$$

In this paper, we adopt the following definition:

$$
D_{*}^{q} x(t)=J^{m-q} x^{(m)}(t), \quad q>0,
$$

where $m=\lceil q\rceil$. The operator $D_{*}^{q}$ is generally called the Caputo differential operator of order $q .{ }^{35,36}$
Consider a fractional-order weighted complex dynamical network consisting of $N$ identical nodes, described by

$$
\begin{gathered}
D_{*}^{q} x_{i}(t)=f\left[x_{i}(t)\right]+c \sum_{j=1, j \neq i}^{N} G_{i j} \Gamma\left[x_{j}(t)-x_{i}(t)\right], \\
i=1,2, \ldots, N,
\end{gathered}
$$

where $0<q \leqslant 1$ is the fractional order; $f(\cdot) \in \mathrm{R}^{n}$ is a given nonlinear continuously differentiable function describing the local dynamics of the nodes; $x_{i}=\left(x_{i 1}, x_{i 2}, \ldots, x_{i n}\right)^{T} \in \mathbb{R}^{n}$ represents the state vector of the $i$ th node; $c>0$ is the overall coupling strength; $\Gamma>0 \in \mathbb{R}^{n \times n}$ is a constant matrix indicating inner-coupling between the elements of the node itself; matrix $G=\left(G_{i j}\right)_{N \times N}$ is the coupling configuration matrix representing the topological structure of the network, if there is a connection between node $i$ and node $j(i \neq j), G_{i j}$ is positive if there is a direct influence from node $j$, where $G_{i j}$ gives a measure of the strength of the interaction. The diagonal elements of matrix $G$ are defined as

$$
G_{i i}=-\sum_{j=1, j \neq i}^{N} G_{i j}
$$

which ensures the diffusion satisfying $\sum_{j=1}^{N} G_{i j}=0$. Suppose that network (5) is connected, then $G$ is an irreducible real matrix, and the network (5) can be rewritten as

$$
D_{*}^{q} x_{i}(t)=f\left[x_{i}(t)\right]+c \sum_{j=1}^{N} G_{i j} \Gamma x_{j}(t), \quad i=1,2, \ldots, N .
$$

In this paper, we focus on a class of weighted fractionalorder networks where $G$ is diagonalizable and has real eigenvalues. In particular, $G$ can be written as

$$
G_{i j}=-\frac{L_{i j}}{k_{i}^{\beta}}
$$

where $k_{i}$ is the degree of node $i$, and $\beta$ is a tunable parameter; the real matrix $L=\left(L_{i j}\right)_{N \times N}$ is the usual (symmetric) Laplacian matrix with diagonal entries $L_{i i}=k_{i}$ and offdiagonal entries $L_{i j}=-1$ if node $i$ and $j$ are connected by a link, and $L_{i j}=0$ otherwise. In this model, we assume that network (7) is strong connected in the sense of having no isolated clusters, which means that the coupling matrix $G$ is irreducible. In sum, $G$ is irreducible and negative semidefine.

Our aim is to stabilize network (7) onto a homogeneous stationary state if

$$
\lim _{t \rightarrow \infty}\left\|x_{i}(t)-\bar{x}\right\|=0, \quad i=1,2, \ldots, N,
$$

where $\bar{x}$ is an equilibrium point satisfying $D_{*}^{q} \bar{x}=f(\bar{x})$; the notation $\|\cdot\|$ represents for the Euclidean vector norm. Our task is to stabilize the networks by using pinning strategy. We apply the pinning control on a small fraction $\sigma(0<\sigma<1)$ of the nodes in the network (7). Without loss of generality, let the first $l$ nodes be controlled as identified by the set $\mathcal{C}=\left\{c_{1}, c_{2}, \ldots, c_{l}\right\}$, where $l=\sigma N$ is the integer part of the real number $\sigma N$.

To achieve the controlling fractional-order network to its equilibriums, one should choose the number of controlled nodes $l$. The decision is influenced by both controllability of 
the network (7) and by selection strategies. A better controllability will probably lead to less cost in terms of control. Moreover, it should be pointed out that applying different strategies for a given network will result in different values of $l$. A smaller $l$ also means less cost. Assume that the set $\mathcal{C}$ contains $l$ nodes where $l$ is a fixed number. As has been shown in Refs. 18-20, 23, and 24, the coupling matrix $G$ and the coupling strength $c$ directly affect the controllability of the network; these parameters also affect the detailed selection, as follows from the above description. Thus, it is difficult to decide on the selection strategy. In the following section, we will show that the fractional-order $q$, the control gain matrix $D$, and the tunable parameter $\beta$ and different pinning techniques also affect the controllability of the network.

\section{STABILITY ANALYSIS}

Before beginning our main results, the following lemmas are needed to derive the main results.

Lemma 1 (see Ref. 37). Consider the following autonomous system:

$$
D_{*}^{q} X=A X, \quad X(0)=X_{0},
$$

with $0<q \leqslant 1, X \in \mathbb{R}^{n}$. System (10) is asymptotically stable if and only if $\left|\arg \left[\lambda_{i}(A)\right]\right|>q \pi / 2, \quad i=1,2, \ldots, n$, where $\arg \left[\lambda_{i}(A)\right]$ denotes the argument of the eigenvalue $\lambda_{i}$ of $A$. In this case, the component of the state decay towards 0 like $t^{-q}$; system (10) is stable if and only if either it is asymptotically stable or those critical eigenvalues which satisfy $\left|\arg \left[\lambda_{i}(A)\right]\right|=q \pi / 2$ have a geometric multiplicity one.

Lemma 2 (see Ref. 22). If $G=\left(G_{i j}\right) \in \mathbb{R}^{N \times N}$ is a real irreducible matrix satisfying the diffusive coupling condition, i.e., $G_{i j} \geqslant 0(j \neq i)$ and $G_{i i}=-\sum_{j=1, j \neq i}^{N} G_{i j}$, then

(i) 0 is the largest eigenvalue of $G$ with multiplicity 1 and $[1,1, \ldots, 1]^{T}$ is the corresponding eigenvector with identical nonzero elements;

(ii) there exists a nonsingular matrix $\Phi$ $=\left[\phi_{1}, \phi_{2}, \ldots, \phi_{N}\right] \in R^{N \times N}$, such that

$$
G \phi_{k}=\lambda_{k} \phi_{k}, \quad k=1,2, \ldots, N,
$$

where $0=\lambda_{1}>\lambda_{2} \geqslant \cdots \geqslant \lambda_{N}$ are the eigenvalues of $G$.

Lemma 2 can be easily proved by the Gerschgorin's disk theorem and the Perron-Frobenius theory.

Lemma 3 (see Ref. 22). If the matrix $G$ is defined as in Lemma 2, and the nonzero diagonal matrix $D$ is defined as $D=\operatorname{diag}\left(d_{1}, d_{2}, \ldots, d_{N}\right)$ with $d_{i} \geqslant 0(i=1,2, \ldots, N)$, then $R$ $=G-D$ is negative definite.

Then, the controlled network (7) can be described as

$D_{*}^{q} x_{i}(t)=f\left[x_{i}(t)\right]+c \sum_{j=1}^{N} G_{i j} \Gamma x_{j}(t)+u_{i}, \quad i=1,2, \ldots, N$,

with the local negative feedback controllers are given by

$$
u_{i}=-c d_{i} \Gamma\left[x_{i}(t)-\bar{x}\right], \quad i=1,2, \ldots, N,
$$

where the feedback gain satisfies

$$
d_{i}=\left\{\begin{array}{l}
d, i=1,2, \ldots, l \\
0, i=l+1, l+2, \ldots, N .
\end{array}\right.
$$

Let the error states be

$$
e_{i}(t)=x_{i}(t)-\bar{x}, \quad i=1,2, \ldots, N .
$$

Then, one can linearize the controlled networks (11) at state $\bar{x}$ to obtain

$$
D_{*}^{q} E(t)=E(t) J^{T}[f(\bar{x})]+c R E(t) \Gamma, \quad i=1,2, \ldots, N,
$$

where $J^{T}[f(\bar{x})]$ is the Jacobian matrix of $f$ evaluated at $\bar{x}$; $E(t)=\left[e_{1}(t), e_{2}(t), \ldots, e_{N}(t)\right]^{T} \in \mathbb{R}^{n \times N}$.

From Lemmas 2 and 3, it follows that $R$ is negative definite, which means that all of its eigenvalues are strictly negative, denoted in an decreasing order as

$$
0>\lambda_{1} \geqslant \lambda_{2} \geqslant \cdots \geqslant \lambda_{N}=\lambda_{\text {min }}(R),
$$

with their corresponding (generalized) eigenvectors $\Phi$ $=\left[\phi_{1}, \phi_{2}, \ldots, \phi_{N}\right] \in \mathbb{R}^{N \times N}, \quad$ satisfying $\quad R \phi_{k}=\lambda_{k} \phi_{k}, \quad k$ $=1,2, \ldots, N$. By expressing each column $E$ on the basis of $\Phi$ basis, one has

$$
E=\Phi \theta
$$

Thus, Eq. (15) can be expanded into the following equations:

$$
D_{*}^{q} \theta_{k}(t)=\left\{J[f(\bar{x})]+c \lambda_{k} \Gamma\right\} \theta_{k}(t), \quad k=1,2, \ldots, N,
$$

where $\theta_{k}(t)=\left[\theta_{k 1}, \theta_{k 2}, \ldots, \theta_{k n}\right]^{T}$.

Now, the local stability problem of the $(N \times n)$-dimensional system $(15)$ is converted into the stability problem of the much simpler $N$ independent $n$-dimensional linear systems (17). One can see that in Eq. (17) only $\lambda_{k}$ and $\theta_{k}$ are related to $k$. The importance of Eq. (17) is that the stability problem of the controlled fractionalorder network (11) can be divided into three independent problems: one is to tune the fractional-order $q$ to make the eigenvalue of matrix $R$ into the stable region; the second is to analyze the stable regions of the fractional-order networks (17), depending on the local dynamics of the isolated node, such as, the equilibrium point $\bar{x}$, the Jacobian matrix $J f(\bar{x})$ and inner coupling matrix $\Gamma$; the last is to analyze the eigenvalue distribution of the matrix $R$, which depends on the network topological structure and control scheme, i.e., the tunable parameter $\beta$, the overall coupling strength $c$, the network size $N$, the coupling matrix $G$ and the control gain matrix $D$.

The following theorem characterizes a necessary and sufficient condition for system (7) to be locally exponentially stable in the homogenous state $\bar{x}$.

Theorem 1: For a certain fractional-order $q$, the controlled network (11) is locally exponentially stable about $\bar{x}$ if and only if all the eigenvalues of the matrix $\left[J f(\bar{x})+c \lambda_{k} \Gamma\right]$ satisfy

$$
\left|\arg \left\{\Lambda_{k i}\left[J f(\bar{x})+c \lambda_{k} \Gamma\right]\right\}\right|>\frac{q \pi}{2},
$$

$$
i=1,2, \ldots, n, \quad k=1,2, \ldots, N,
$$

where $\Lambda_{k i}$ is the eigenvalue of the $k$ th node matrix $\left[J f(\bar{x})+c \lambda_{k} \Gamma\right]$.

Proof: By using Lemma 1, Theorem 1 can be easily proved. 
Corollary 1: For certain $\lambda_{k}$, inner coupling matrix $\Gamma$, overall coupling strength $c$, the control gain $D$, and the coupling matrix $G$, the controlled network (11) is locally exponentially stable about $\bar{x}$ if and only if all the eigenvalues of the matrix $\left[J f(\bar{x})+c \lambda_{k} \Gamma\right]$ satisfy

$$
\begin{aligned}
& q<\frac{2}{\pi} \tan ^{-1}\left|\frac{\left.\operatorname{Im}\left\{\Lambda_{k i}\left[J f(\bar{x})+c \lambda_{k} \Gamma\right]\right\}\right)}{\left.\operatorname{Re}\left\{\Lambda_{k i}\left[J f(\bar{x})+c \lambda_{k} \Gamma\right]\right\}\right)}\right|, \\
& i=1,2, \ldots, n, \quad k=1,2, \ldots, N .
\end{aligned}
$$

Remark 1: One can easily see that Corollary 1 is essentially equivalent to Theorem 1 . However, the significance of Corollary 1 is to show us that reducing the value of fractional-order $q$ can stabilize the homogenous stationary state when the coupling matrix $G$, the control gain matrix $D$, the coupling strength $c$, the inner matrix $\Gamma$, the pinning schemes are fixed all the time. It can be seen that the stable region is becoming larger, while the unstable region is becoming smaller. Corollary 1 gives us a new idea that we can adjust the fractional-order $q$ to achieve stabilization. It should be noted that even if the networks may not be pinned by any node, the networks can also be stabilized by tuning fractional-order $q$. One can see the numerical simulation in Sec. IV C which supports this point.

Hereafter, we give a more useful theorem to characterizes a necessary and sufficient condition for system (11) to be locally exponentially stable in the homogenous state $\bar{x}$.

Theorem 2: For certain $\lambda_{k}$, inner coupling matrix $\Gamma$, overall coupling strength $c$, the control gain $D$, and the coupling matrix $G$, the controlled network (11) is locally exponentially stable about $\bar{x}$ if and only if all the eigenvalues of the matrix $\left[J f(\bar{x})+c \lambda_{1} \Gamma\right]$ satisfy $\left|\arg \left\{\Lambda_{1 i}\left[J f(\bar{x})+c \lambda_{1} \Gamma\right]\right\}\right|$ $>q \pi / 2, i=1,2, \ldots, n$.

Proof: Since $\lambda_{1}$ is the largest eigenvalue of the negative definite matrix $R$ [see Eq. (15)], only if the eigenvalues of the matrix $\left[J f(\bar{x})+c \lambda_{1} \Gamma\right]$ satisfy $\left|\arg \left\{\Lambda_{1 i}\left[J f(\bar{x})+c \lambda_{1} \Gamma\right]\right\}\right|>q \pi / 2$, all the eigenvalues of $\left[J f(\bar{x})+c \lambda_{k} \Gamma\right], k=2, \ldots, N$ must satisfy the Lemma 1. Thus, according to the stability theory of fractional-order system, the controlled network (11) is locally exponentially stable about $\bar{x}$. The necessity is obvious. This completes the proof.

Corollary 2: For certain $\lambda_{k}$, inner coupling matrix $\Gamma$, overall coupling strength $c$, the control gain $D$, and the coupling matrix $G$, the controlled network (11) is locally exponentially stable about $\bar{x}$ if and only if all the eigenvalues of the matrix $\left[J f(\bar{x})+c \lambda_{1} \Gamma\right]$ satisfy

$q<\frac{2}{\pi} \tan ^{-1}\left|\frac{\left.\operatorname{Im}\left\{\Lambda_{1 i}\left[J f(\bar{x})+c \lambda_{1} \Gamma\right]\right\}\right)}{\left.\operatorname{Re}\left\{\Lambda_{1 i}\left[J f(\bar{x})+c \lambda_{1} \Gamma\right]\right\}\right)}\right|, \quad i=1,2, \ldots, n$.

Suppose that there exists a constant $\rho>0$ such that its eigenvalues of the matrix $[J f(\bar{x})-\rho \Gamma]$ satisfy $\mid \arg \left\{\Lambda_{1 i}[J f(\bar{x})\right.$ $-\rho \Gamma]\} \mid>q \pi / 2, i=1,2, \ldots, n$. If $c \lambda_{1} \leqslant-\rho$, then $c \lambda_{k} \leqslant-\rho$ for $k=1,2, \ldots, N$. Thus, all the eigenvalues of $\left[J f(\bar{x})+c \lambda_{k} \Gamma\right]$ have satisfied Lemma 1 . Then, the following corollary can be obtained without any difficulty.

Corollary 3: Assume that there is a constant $\rho>0$ such that all the eigenvalues of the matrix $[J f(\bar{x})-\rho \Gamma]$ satisfy $\left|\arg \left\{\Lambda_{1 i}[J f(\bar{x})-\rho \Gamma]\right\}\right|>q \pi / 2, i=1,2, \ldots, n$. Then, the controlled network (11) is locally exponentially stable about $\bar{x}$, provided that

$$
c \lambda_{1} \leqslant-\rho .
$$

By the idea of pinning control, the number of controllers is preferred to be very small compared with the entire network size $N$, namely, $l \ll N$. According to Lemma $2, R$ is negative if $D \neq 0$. Considering the best possible case of $l$ $=1$, we can easily obtain the following result from the above analysis.

Corollary 4: Suppose that the feedback gain matrix $D=\operatorname{diag}(d, 0, \ldots, 0)$, which means that only one node is to be pinned. Meanwhile, we assume that there exists a constant $\rho>0$ as in Corollary 1 such that all the eigenvalues of $[J f(\bar{x})-\rho \Gamma]$ satisfy $\left|\arg \left\{\Lambda_{1 i}[J f(\bar{x})-\rho \Gamma]\right\}\right|>q \pi / 2$, $i=1,2, \ldots, n$. Then the controlled network (11) is locally exponentially stable about $\bar{x}$, provided that

$$
c \lambda_{1} \leqslant-\rho .
$$

Remark 2: Obviously, one can see that Corollary 4 is a special case of Corollary 3. The importance of Corollary 4 is to tell us that pinning any one node in the network can stabilize the homogenous stationary state when the network is controllable and connected all the time. From the conditions (21) and (22), it follows that fixed coupling matrix $G$, control gain matrix $D$, and the overall coupling strength $c$ guarantee the fractional-order network stabilization.

We can easily obtain the following result from the above analysis.

Corollary 5: Suppose that the feedback gain matrix $D=\operatorname{diag}(d, 0, \ldots, 0)$, which means that only one node is to be pinned. Moreover, we assume that there exists a constant $\rho>0$ as in Corollary 1 such that all the eigenvalues of $[J f(\bar{x})-\rho \Gamma]$ satisfy $\left|\arg \left\{\Lambda_{1 i}[J f(\bar{x})-\rho \Gamma]\right\}\right|>q \pi / 2$, $i=1,2, \ldots, n$. Then the controlled network (11) is locally exponentially stable about $\bar{x}$, provided that

$$
c \geqslant-\rho / \lambda_{1}>0 \text {. }
$$

The Corollary 5 shows that sufficiently strong overall coupling strength will lead a network to stabilized.

On the other hand, the following result can be obtained.

Corollary 6: Suppose that the feedback gain matrix $D=\operatorname{diag}(d, 0, \ldots, 0)$, which means that only one node is to be pinned. Moreover, we assume that there exists a constant $\rho>0$ as in Corollary 1 such that all the eigenvalues of $[J f(\bar{x})-\rho \Gamma]$ satisfy $\left|\arg \left\{\Lambda_{1 i}[J f(\bar{x})-\rho \Gamma]\right\}\right|>q \pi / 2$, $i=1,2, \ldots, n$. Then the controlled network (11) is locally exponentially stable about $\bar{x}$, provided that

$$
\lambda_{1} \leqslant-\rho / c<0 \text {. }
$$

Remark 3: It is can be revealed from Corollary 6 that, for a given coupling strength $c$ and the coupling matrix $G$, we can choose control gain matrix $D$ (i.e., choose sufficiently large $l$ or large $d$ ) to stabilize the network to its equilibriums.

\section{EXAMPLES}

In this section, in order to show the effectiveness of the proposed results, some numerical examples in scale-free 
complex networks are provided by employing different pinning schemes. Moreover, it is worth mentioning that some properties of fractional-order complex networks, such as, the fractional-order $q$, the tunable parameter $\beta$, the overall coupling strength $c$, the coupling matrix $G$, the control gain matrix $D$, and different pinning schemes, will affect the controllability of weighted fractional-order networks.

\section{A. Numerical algorithms}

In order to simulate the fractional-order complex networks, we have to make some modifications of the algorithms proposed in Ref. 38. The algorithms to simulate the fractional-order complex networks without control can be given as follows:

The fractional-order differential equations with coupling terms are given by

$$
\begin{aligned}
& D_{*}^{q} x_{i}(t)=f\left[t, x_{i}(t)\right]+\sum_{j=1}^{N} G_{i j} \Gamma x_{j}(t), 0 \leqslant t \leqslant T, \\
& x_{i}^{(k)}(0)=x_{i 0}^{(k)}, \quad k=0,1, \ldots, m-1, \quad i=1,2, \ldots, N,
\end{aligned}
$$

where $q, m$ are defined in Eq. (1) and $N$ is the size of complex networks. This differential equation with coupling terms is equivalent to Volterra integral equation

$$
x_{i}(t)=\sum_{k=0}^{\lceil q\rceil-1} \frac{t^{k}}{k !} x_{i 0}^{(k)}+\frac{\int_{0}^{t}(t-\tau)^{q-1}\left\{f\left[\tau, x_{i}(\tau)\right]+\sum_{j=1}^{N} G_{i j} \Gamma x_{j}(\tau)\right\} d \tau}{\Gamma(q)} .
$$

Set $h=T / W, W \in Z, t_{n}=n h,(n=0,1, \ldots, W)$, where $h$ is the step size, $T$ is simulation time, and $W$ is the number of sample points. Then, Eq. (26) can be discretized as follows:

$$
\begin{aligned}
x_{i h}\left(t_{n+1}\right)= & \sum_{k=0}^{\lceil q\rceil-1} \frac{t_{n+1}^{k}}{k !} x_{i 0}^{(k)}+\frac{h^{q}\left\{f\left[t_{n+1}, x_{i h}^{p}\left(t_{n+1}\right)+\sum_{j=1}^{N} G_{i j} \Gamma x_{j h}\left(t_{n+1}\right)\right]\right\}}{\Gamma(q+2)}+\frac{h^{q}}{\Gamma(q+2)} \sum_{l=0}^{n} a_{l, n+1}\left(\left\{f\left[t_{l}, x_{i h}\left(t_{l}\right)\right]+\sum_{j=1}^{N} G_{i j} \Gamma x_{j h}\left(t_{l}\right)\right\}\right), \\
& i=1,2, \ldots, N,
\end{aligned}
$$

where

$$
\begin{aligned}
& a_{l, n+1}=\left\{\begin{array}{ll}
n^{q+1}-(n-q)(n+1)^{q}, & \text { if } l=0 \\
(n-l+2)^{q+1}+(n-l)^{q+1}-2(n-l+1)^{q+1}, & \text { if } 1 \leqslant l \leqslant n \\
1, & \text { if } l=n+1
\end{array}\right\}, \\
& x_{i h}^{p}\left(t_{n+1}\right)=\sum_{k=0}^{\lceil q]-1} \frac{t_{n+1}^{k}}{k !} x_{i 0}^{(k)}+\frac{1}{\Gamma(q)} \sum_{l=0}^{n} b_{l, n+1}\left\{f\left[t_{l}, x_{i h}\left(t_{l}\right)+\sum_{j=1}^{N} G_{i j} \Gamma x_{j h}\left(t_{l}\right)\right]\right\},
\end{aligned}
$$

where $b_{l, n+1}=h^{q}\left[(n+1-l)^{q}-(n-l)^{q}\right] / q$.

Therefore, the error estimate is

$$
\max _{s=0,1, \ldots, N}\left|x\left(t_{s}\right)-x_{h}\left(t_{s}\right)\right|=O\left(h^{p}\right),
$$

where $p=\min (2,1+q)$.

Remark $4:$ In order to simulate the fractional-order system, the simulation algorithm proposed in this paper needs to be effective and solvable. However, if the networks size $N$ and sampled point $W$ are very large, the procedure usually needs large-scale computation and data processing. Therefore, it is still a challenging problem to solve the optimization problem with low computational complexity.

\section{B. Eigenvalues distribution analysis}

In 2002, Lü et al. ${ }^{39}$ introduced a new chaotic system which can unify chaotic systems, such as, the Lorenz, Chen, Lü systems. The fractional-order differential equation of the unified system can be given by

$$
\begin{aligned}
& \frac{d^{q} x}{d t^{q}}=(25 \alpha+10)(y-x), \\
& \frac{d^{q} y}{d t^{q}}=(28-35 \alpha) x-x z+(29 \alpha-1) y, \\
& \frac{d^{q} z}{d t^{q}}=x y-\frac{\alpha+8}{3} z,
\end{aligned}
$$

where $0<q \leqslant 1, \alpha \in[0,1]$. When $\alpha=0, \alpha=0.8, \alpha=1$, and $q=1$, the unified integer-order chaotic system is the Lorenz, Lü, and Chen system, respectively. The chaotic behaviors can be seen from Figs. 1 and 2 the chaotic behaviors when $q=0.99, \alpha=0$, and $q=0.9, \alpha=1$, respectively.

For the chaotic system in the continuous time domain, there exists at least one eigenvalue of $J f(\bar{x})$, which does not satisfy Lemma 1. The goal is to ensure all the eigenvalues of $\Lambda_{1 i}\left[J f(\bar{x})+c \lambda_{1} \Gamma\right]$ are well located in the stable region through designing the feedback gain matrix in the case of fixed topology. Here, we aim at investigating that the Lorenz system is taken as the isolated node to illustrate the theoretical result. With this set of system parameters, the unstable equilibrium points are $\bar{x}=[ \pm 6 \sqrt{2}, \pm 6 \sqrt{2}, \pm 27]^{T}$ and 


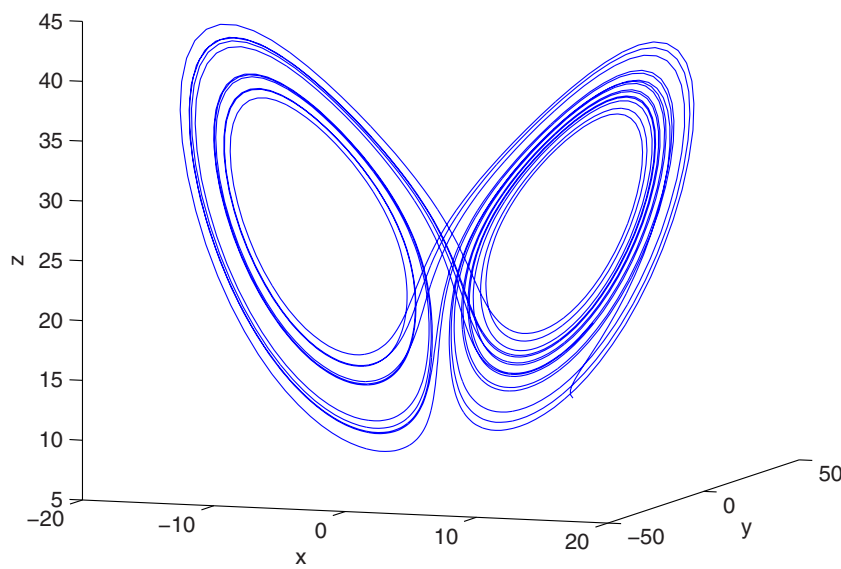

FIG. 1. (Color online) Chaotic attractor of Lorenz oscillator with $q=0.99$, $\alpha=0$, and initial vector $[x(0), y(0), z(0)]=(10,3,12)$.

$\bar{x}=[0,0,0]^{T}$. Here, we stabilize the controlled networks to $\bar{x}=[6 \sqrt{2}, 6 \sqrt{2}, 27]^{T}$. It is an easy matter to obtain the eigenvalue distribution of $J f(\bar{x})$ as shown in Fig. 3. The objective is to make two of the eigenvalues in the unstable region cross the critical line and enter the stable region by adding an appropriate control matrix $D$.

In the simulation, the size of the scale-free network is $N=20$. The model can be described as follows. The growth starts from with three nodes and no edges. At each step, a new node with three edges is added to the existing network. Repeating this rule will produce a scale-free networks. In the following, we choose $G_{i j}=L_{i j} / k_{i}^{\beta}$, where $\beta$ is a tunable parameter, $L$ is the Laplacian matrix of the network. Consider a scale-free network composed of $N=20$ identical Lorenz oscillators in the case of $c=10$ and $\Gamma=\operatorname{diag}(1,1,1)$. We select only one of the nodes to be pinned and set the feedback gain $d$ as 10 . The fractional-order $q$ is chosen as $q=0.99$ and the tunable parameter $\beta=0.1$. As can be seen in Fig. 4, two eigenvalues are in the unstable region (as in Fig. 3), and then they move across the line and enter the stable region. This suggests that the feedback gain designed is efficient for stability.

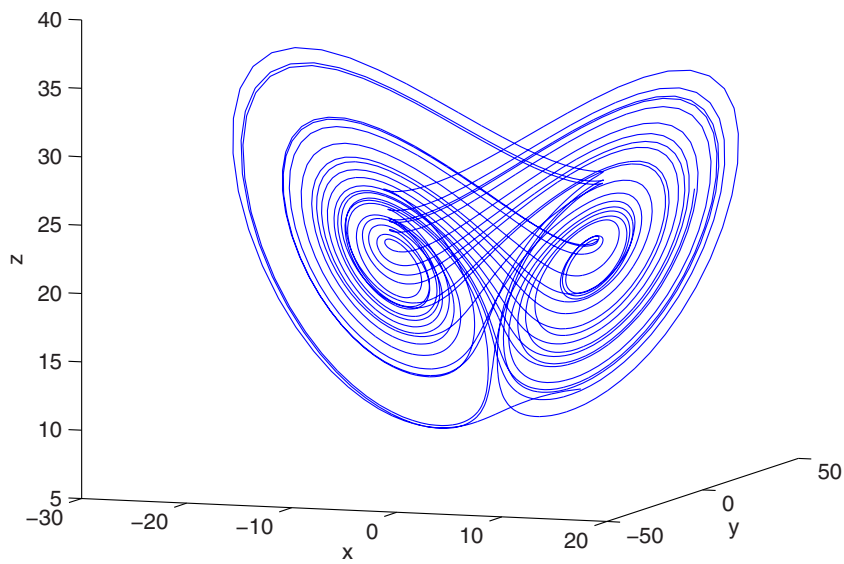

FIG. 2. (Color online) Chaotic attractor of Chen oscillator with $q=0.90$, $\alpha=1$, and initial vector $[x(0), y(0), z(0)]=(10,3,12)$.

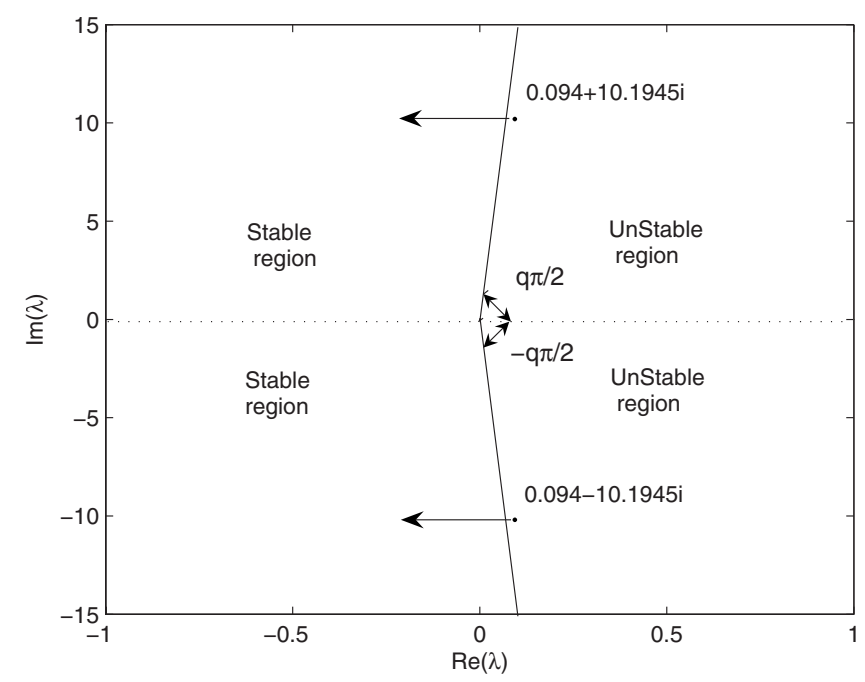

FIG. 3. Eigenvalue distribution of the Jacobian matrix $J f(\bar{x})$. One of the eigenvalues located in the stable region is omitted.

\section{Pinning by adjusting fractional-order $q$}

Here, we consider a scale-free network and Lorenz system discussed in Sec. IV B. The pinning scheme is employed to pin only one node of the networks. We set $c=10, \beta=0.1$, $d=10$ and only tuning fractional-order $q$. Note that we still select the same single node to control the network while adjusting the fractional-order $q$. For the sake of pinning scheme, we introduce a quantity

$$
E(t)=\sqrt{\frac{\sum_{i=1}^{N}\left\|x_{i}(t)-\bar{x}\right\|^{2}}{N}},
$$

which is used to measure the quality of the pinning process. From Fig. 5, one can easily see that the control performance is better with the decreasing of fractional-order $q$. As seen in Lemma 1, Figs. 3 and 4, when the fractional-order $q$ decreases, the stable region expands, while the unstable region becomes smaller.

Remark 5: Surprisingly, from Corollary 1, if any node of

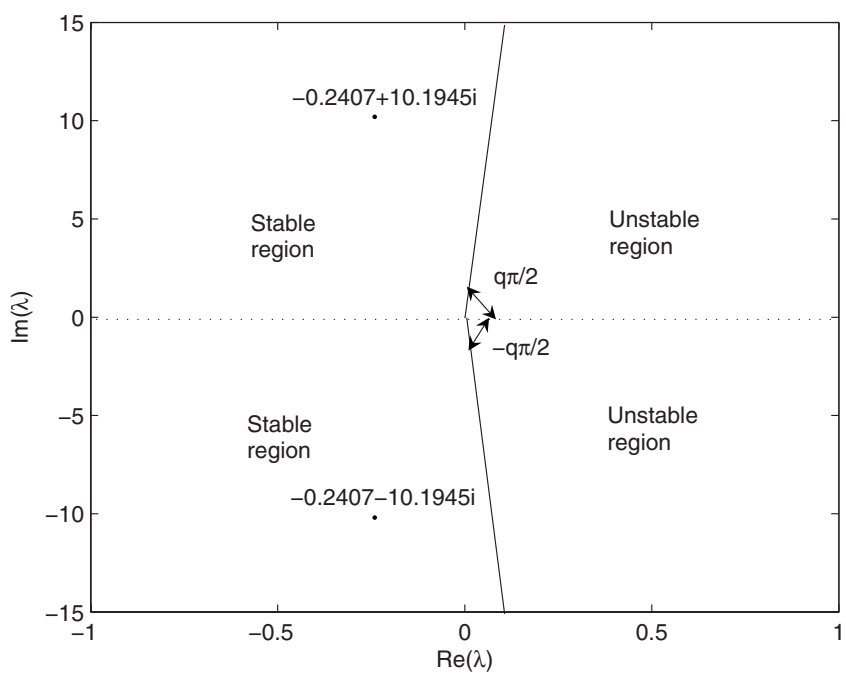

FIG. 4. Eigenvalue distribution of matrix $J f(\bar{x})+c \lambda_{1} \Gamma$. One of the eigenvalues located in the stable region is omitted. 


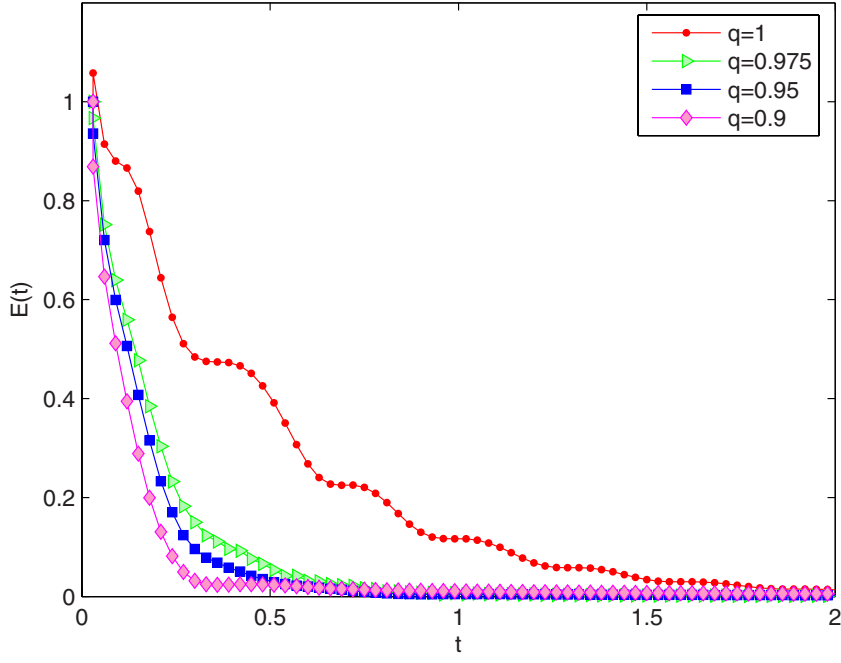

FIG. 5. (Color online) The quantity $E(t)$ of pinning a certain node in scalefree network under different fractional-order $q$.

the networks is not pinned, the fractional-order networks can stabilize itself without any controllers, while $q$ should satisfy $q<0.9941$. It is can be seen from Fig. 6 that, after $2.5 \mathrm{~s}$, the fractional-order networks is stable without control at its equilibrium, where $q=0.9$. On the other hand, the results in this paper can be easily extended to other chaotic systems, such as, the Chua system, hyperchaotic systems, and so on.

\section{Pinning by adjusting control gain matrix $D$}

We consider the same scale-free network discussed in Sec. IV B and Chen system as each node. The pinning scheme is employed to pin one node of the networks. Let $c$ $=10, \beta=0.5, q=0.90$, and only tuning control gain matrix $D=\operatorname{diag}(d, 0,0, \ldots, 0)$. Note that we still select the same single node to control the network. We also use Eq. (30) to evaluate the control performance. Therefore, only the control gain of one node is changed. From Fig. 7, one can easily see that the control performance is better with the increase of control gain $d$.

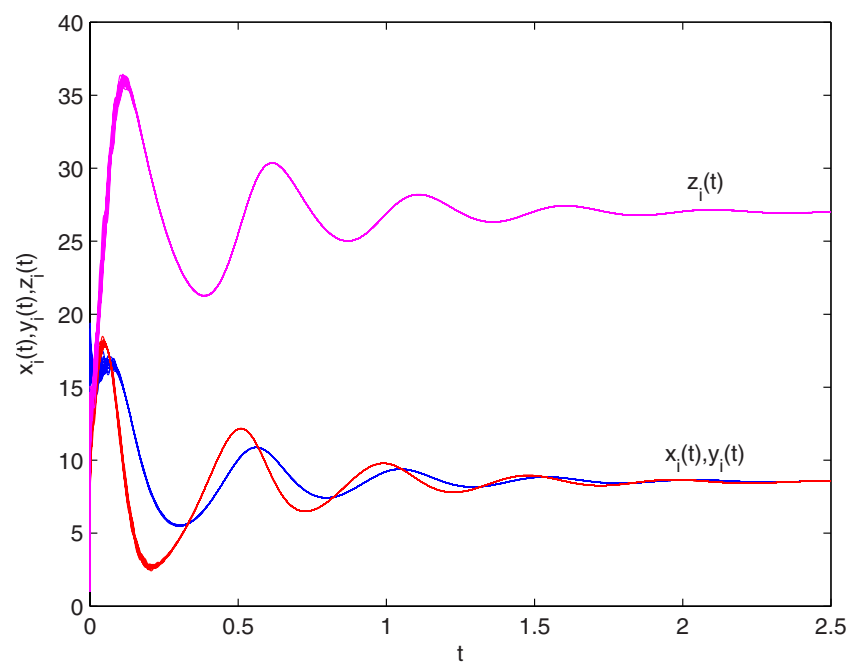

FIG. 6. (Color online) Convergence of states in the scale-free network under $q=0.9$ without pinning any node.

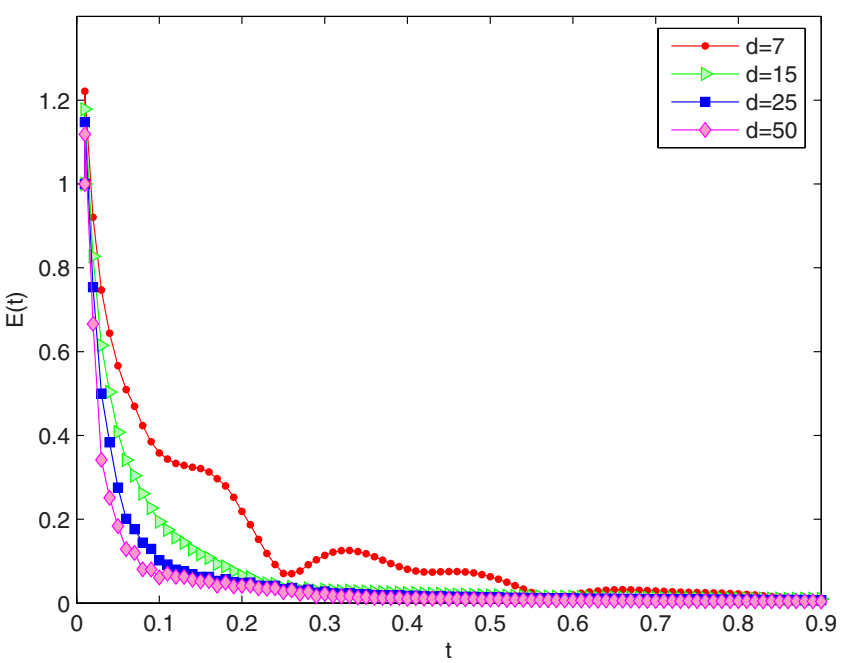

FIG. 7. (Color online) The quantity $E(t)$ of pinning a certain node in the scale-free network under different control gain $d$.

\section{E. Pinning by tuning overall coupling strength $c$}

Consider the same scale-free network discussed in Sec. IV B and Chen system as each node. The pinning scheme is applied to pin the nodes of the networks. Let $\beta=0.5, q$ $=0.90, d=10$ and only tuning the overall coupling strength $c$. Notice that we still select the same single node to control the network. Clearly, from Fig. 8, we can easily see that the control performance is better with the increase of overall coupling strength $c$.

\section{F. Pinning by adjusting tunable parameter $\beta$}

Consider the same scale-free network discussed in Sec. IV B and Chen system as each node. The pinning scheme is used to pin one node of the networks. Let $c=10, q=0.95$, $d=10$, and only change the tunable parameter $\beta$. It should be emphasized that we still select the same single node to control the network. $\beta=0$ indicates that the coupling matrix $G$ is an unweighted symmetric matrix. Obviously, from Fig. 9, we can easily see that the control performance is better, while

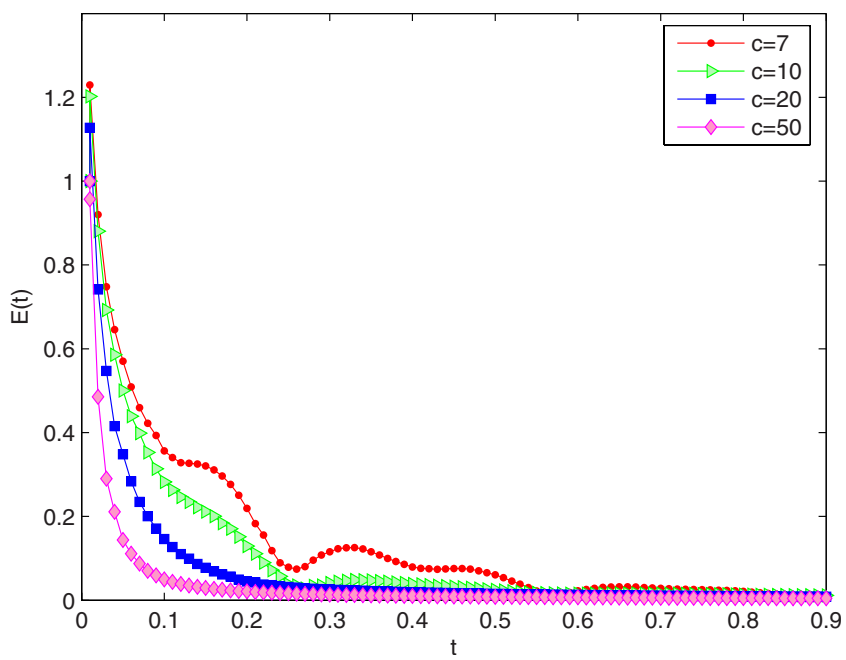

FIG. 8. (Color online) The quantity $E(t)$ of pinning a certain node in the scale-free network under different coupling strength $c$. 


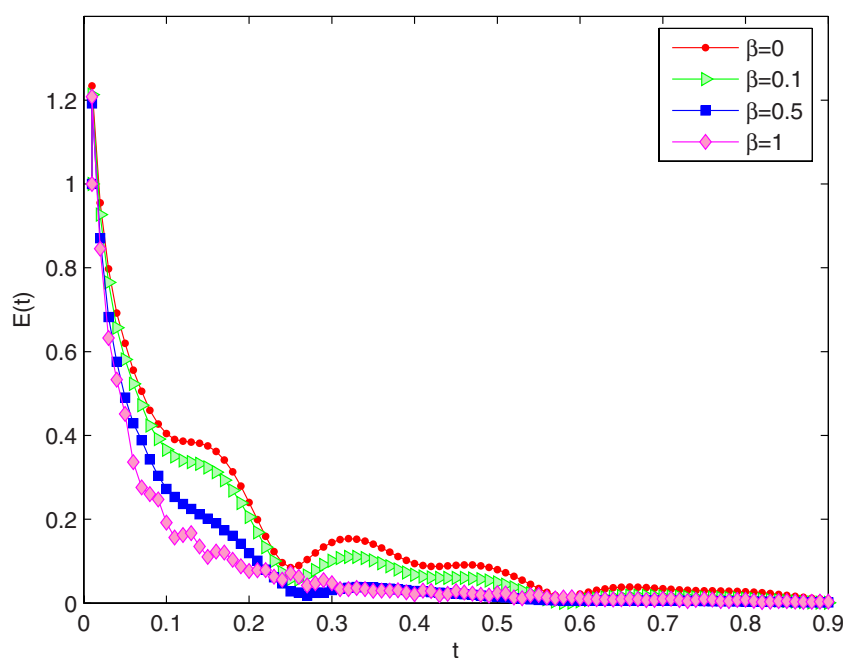

FIG. 9. (Color online) The quantity $E(t)$ of pinning a certain node in the scale-free network under different parameters $\beta$.

parameter $\beta$ is increasing. The simulation shows that a fractional-order complex network-weighted or unweighted, symmetric or asymmetric-can be stabilized through pinning.

\section{G. Randomly pinning and specially pinning}

Consider the same scale-free network discussed in Sec. IV B and Chen system as each node. Two different pinning schemes are used to pin the nodes of the networks. Let $c$ $=10, q=0.95, d=10, \beta=0.5$ and keep constant. First, only one largest degree node is pinned which has degree 13. Second, the two largest degree nodes are pinned which have degree 13 and 11, respectively. Finally, the randomly pinning scheme is used to pin only one node and two nodes, respectively. Obviously, we can easily see that the control performance is better by pinning a larger degree node and more forceful nodes from Fig. 10.

Remark 6: Clearly, increasing the pinning fraction $\sigma$ will accelerate the convergence of the network stabilization, as shown in Fig. 10. In other words, the more forcefully the

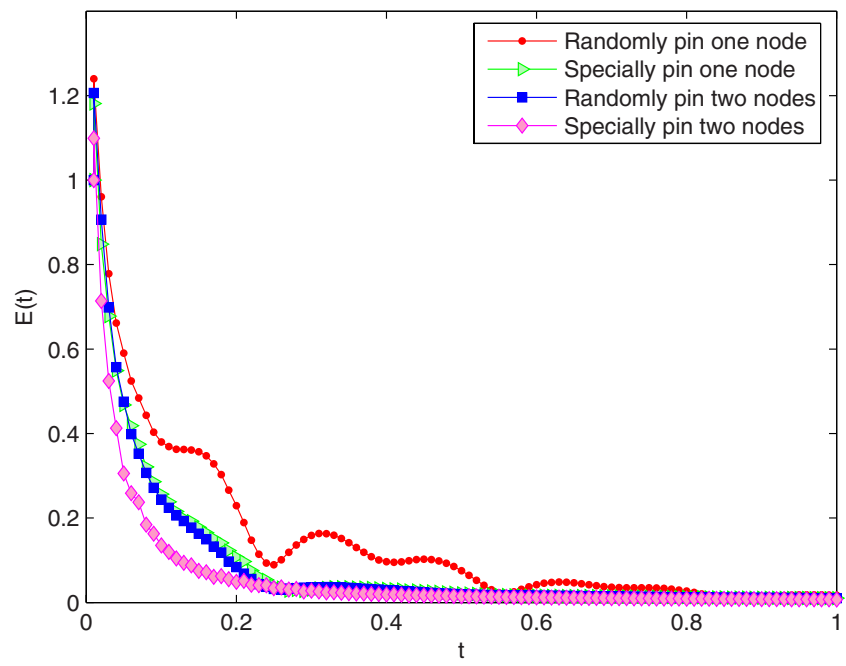

FIG. 10. (Color online) The quantity $E(t)$ of pinning one or two certain nodes in the scale-free network under different pinning schemes. nodes are pinned, the quicker the network is stabilized. On the other hand, in a scale-free network, to reach the same control efficiency, much larger coupling strength and pinning fraction are required in the randomly pinning control than that in the specifically pinning control. That is to say, the randomly pinning scheme is less efficient than the specifically pinning scheme, as compared in Fig. 10.

\section{CONCLUSIONS}

The pinning control problem of a class of fractionalorder weighted complex dynamical networks has been investigated in detail. The general strategy is to apply a feedback control scheme to a small fraction of the network nodes. By utilizing eigenvalue analysis approach and fractional-order stability theory, we employ the numerical algorithms for fractional-order complex networks addressed in this paper to establish some local stability criteria and valid stability regions of such pinned fractional-order networks. The analytical analysis show that the largest eigenvalue of matrix $R$ determines the control of the weighted fractional-order complex networks. By seeking an appropriate $R$, overall coupling strength $c$, and fractional-order $q$, we are able to achieve our goal. It is surprising to find that a network can realize stabilization under a suitable $q$, even without controller. In addition, it is interesting to find that the fractional-order $q$, the control gain matrix $D$, the tunable weight parameter $\beta$, the overall coupling strength $c$, the specially pinning of largest nodes will effectively affect the convergence rate of controlling fractional-order complex dynamical networks. In the end of the paper, some simulation examples in scale-free networks are exploited to demonstrate the applicability of the proposed results.

\section{ACKNOWLEDGMENTS}

The authors are grateful to the editor and reviewers for their kind help and constructive comments which helped to improve the presentation of the paper.

This research was supported in part by the National Natural Science Foundation of P. R. China under Grant No. 60874113, the Key Creative Project of Shanghai Education Community (09ZZ66), the Research Fund for the Doctoral Program of Higher Education (200802550007), and Shanghai Natural Science Foundation (08ZR1400400), the Engineering and Physical Sciences Research Council (EPSRC) of the U.K. under Grant No. GR/S27658/01, an International Joint Project sponsored by the Royal Society of the U.K., and the Alexander von Humboldt Foundation of Germany.

${ }^{1}$ D. J. Watts and S. H. Strogatz, Nature (London) 393, 440 (1998).

${ }^{2}$ A. L. Barabási and R. Albert, Science 286, 509 (1999).

${ }^{3}$ S. H. Strogatz, Nature (London) 410, 268 (2001).

${ }^{4}$ S. Boccalettia, V. Latorab, Y. Morenod, M. Chavezf, and D. U. Hwanga, Phys. Rep. 424, 175 (2006).

${ }^{5}$ V. Latora and M. Marchiori, Phys. Rev. Lett. 87, 198701 (2001).

${ }^{6}$ C. Zhou, A. E. Motter, and J. Kurths, Phys. Rev. Lett. 96, 034101 (2006).

${ }^{7}$ X. F. Wang and G. Chen, Physica A 310, 521 (2002).

${ }^{8}$ M. Barahona and L. M. Pecora, Phys. Rev. Lett. 89, 054101 (2002).

${ }^{9} \mathrm{R}$. Hilfer, Applications of Fractional Calculus in Physics (World Scientific, Singapore, 2000).

${ }^{10}$ I. Podlubny, Fractional Differential Equations (Academic, New York, 1999). 
${ }^{11}$ S. G. Samko, A. A. Kilbas, and O. I. Marichev, Fractional Integrals and Derivatives: Theory and Applications (Gordon and Breach, Yverdon, 1993).

${ }^{12}$ W. Deng and J. Lü, Chaos 16, 043120 (2006).

${ }^{13}$ I. Grigorenko and E. Grigorenko, Phys. Rev. Lett. 91, 034101 (2003).

${ }^{14}$ T. T. Hartley, C. F. Lorenzo, and H. K. Qammer, IEEE Trans. Circuits Syst., I: Fundam. Theory Appl. 42, 485 (1995).

${ }^{15}$ P. Arena, L. Fortuna, and D. Porto, Phys. Rev. E 61, 776 (2000).

${ }^{16} \mathrm{C}$. Li and G. Chen, Physica A 341, 55 (2004).

${ }^{17}$ R. O. Grigoriev, M. C. Cross, and H. G. Schuster, Phys. Rev. Lett. 79, 2795 (1997).

${ }^{18}$ X. Li, X. F. Wang, and G. Chen, IEEE Trans. Circuits Syst., I: Regul. Pap. 51, 2074 (2004).

${ }^{19}$ Q. Miao, Z. H. Rong, Y. Tang, and J. A. Fang, Physica A 387, 6225 (2008).

${ }^{20}$ J. Zhou, J. Lu, and J. Lü, Automatica 44, 996 (2008).

${ }^{21}$ C. Zhou and J. Kurths, Phys. Rev. Lett. 96, 164102 (2006).

${ }^{22}$ L. Y. Xiang, Z. X. Liu, Z. Q. Chen, F. Chen, and Z. Z. Yuan, Physica A 379, 298 (2007).

${ }^{23}$ F. Sorrentino, M. di Bernardo, F. Garofalo, and G. Chen, Phys. Rev. E 75, 046103 (2007).

${ }^{24}$ T. P. Chen, X. W. Liu, and W. L. Lu, IEEE Trans. Circuits Syst., I: Regul. Pap. 54, 1317 (2007).

${ }^{25}$ M. Chavez, D.-U. Hwang, A. Amann, H. G. E. Hentschel, and S. Boccaletti, Phys. Rev. Lett. 94, 218701 (2005).
${ }^{26}$ W. Yu, G. Chen, and J. Lü, Automatica 45, 429 (2009).

${ }^{27}$ L. Y. Xiang, Z. X. Liu, Z. Q. Chen et al., J. Control Theory Appl. 6, 2 (2008).

${ }^{28} \mathrm{C}$. W. Wu and L. O. Chua, IEEE Trans. Circuits Syst., I: Fundam. Theory Appl. 42, 430 (1995)

${ }^{29}$ Y. Liu, Z. Wang, and X. Liu, Phys. Lett. A 372, 3986 (2008).

${ }^{30}$ Y. Liu, Z. Wang, J. Liang, and X. Liu, IEEE Trans. Syst., Man, Cybern., Part B: Cybern. 38, 1314 (2008)

${ }^{31}$ Y. Tang, R. Qiu, J. A. Fang, Q. Miao, and M. Xia, Phys. Lett. A 372, 4425 (2008)

${ }^{32}$ Y. Tang and J. A. Fang, Phys. Lett. A 372, 1816 (2008).

${ }^{33}$ L. Wang, H. P. Dai, H. Dong, Y. Y. Cao, and Y. X. Sun, Eur. Phys. J. B 61 335 (2008).

${ }^{34}$ L. M. Pecora and T. L. Carroll, Phys. Rev. Lett. 80, 2109 (1998).

${ }^{35}$ M. Caputo, Geophys. J. R. Astron. Soc. 13, 529 (1967).

${ }^{36}$ F. Keil, W. Mackens, and J. Werther, Scientific Computing in Chemical Engineering II-Computational Fluid Dynamics, Reaction Engineering, and Molecular Properties (Springer-Verlag, Heidelberg, 1999).

${ }^{37}$ K. Diethelm K, N. J. Ford, and A. D. Freed, Nonlinear Dyn. 29, 3 (2002)

${ }^{38} \mathrm{D}$. Matignon, Stability results of fractional differential equations with applications to control processing, in: IMACS, IEEE-SMC, Lille, France, 1996.

${ }^{39}$ J. Lü and G. Chen, Int. J. Bifurcation Chaos Appl. Sci. Eng. 12, 659 (2002) 